PROCEEDINGS OF THE

AMERICAN MATHEMATICAL SOCIETY

Volume 126, Number 1, January 1998, Pages 123-125

S $0002-9939(98) 04354-8$

\title{
SUFFICIENT CONDITIONS FOR THE OSCILLATION OF FORCED SUPER-LINEAR SECOND ORDER DIFFERENTIAL EQUATIONS WITH OSCILLATORY POTENTIAL
}

\author{
A. H. NASR
}

(Communicated by Hal L. Smith)

\begin{abstract}
In the case of oscillatory potentials, we give sufficient conditions for the oscillation of the forced super-linear equation

$$
x^{\prime \prime}(t)+a(t)|x(t)|^{\nu} \operatorname{sgn} x(t)=g(t) .
$$
\end{abstract}

This answers a question raised by J. S. W. Wong.

\section{INTRODUCTION}

Consider the forced nonlinear second order differential equation

$$
x^{\prime \prime}(t)+a(t)|x(t)|^{\nu} \operatorname{sgn} x(t)=g(t),
$$

where $t \in \mathbb{R}_{+}=[0, \infty), a(t), g(t)$ are real-valued piecewise continuous functions defined on $\mathbb{R}_{+}$and $\nu>1$.

In the case of $\nu>1$, equation (1) is the prototype of a wide class of nonlinear equations called super-linear equations. A solution of (1) is said to be oscillatory if it is defined on some ray $[\alpha, \infty)$ and has an unbounded set of zeros. The equation will be called oscillatory if every solution on some ray is oscillatory. In [7] one can find different conditions sufficient for the oscillation of all solutions of equation (1) assuming that $a(t) \geq 0$. The technique of the proof depends mainly on the assumption, made earlier by Kartsatos (see [3], [4]), that there exists an oscillatory function $h(t)$ such that $h^{\prime \prime}(t)=g(t)$. Other results on forced oscillations of nonlinear second order equations of type (1) can be found in [5] and for linear equations in [6]. It is worthy to notice that the oscillaton of forced linear equations with oscillatory potentials were treated in [2].

In the present paper, we give conditions sufficient for the existence of a zero for every solution of (1) in some interval $[a, b]$. This result is used to answer a question raised by J. S. W. Wong [7, p. 673] about sufficient conditions for the oscillation of forced nonlinear equations in the case the potential $a(t)$ is oscillatory.

Received by the editors March 19, 1996.

1991 Mathematics Subject Classification. Primary 34C15.

Key words and phrases. Nonlinear second order differential equations, oscillation.

The author is deceased.

(C) 1998 American Mathematical Society 


\section{THE MAIN RESUlT}

Consider equation (1) in the case $\nu>1$. Assume that there exists an interval $[a, b] \subset \mathbb{R}_{+}$such that

$$
a(t) \geq 0 \text { for all } t \in[a, b] .
$$

Moreover, there exists $c \in(a, b)$ such that $g(t)$ has different signs on $[a, c]$ and $[c, b]$. For instance, let $g(t) \leq 0$ on $[a, c]$ and $g(t) \geq 0$ on $[c, b]$.

Theorem. Assume that there exists a continuous function $u(t)$ such that $u(a)=$ $u(b)=u(c)=0, u(t)$ is differentiable on the open set $(a, c) \cup(c, b)$ and

$$
\begin{aligned}
& \int_{a}^{c}\left[a^{1 / \nu}(t)|g(t)|^{1-1 / \nu} u^{2}(t)-{u^{\prime}}^{2}(t)\right] d t \geq 0, \\
& \int_{c}^{b}\left[a^{1 / \nu}(t)|g(t)|^{1-1 / \nu} u^{2}(t)-u^{\prime 2}(t)\right] d t \geq 0 .
\end{aligned}
$$

Then every solution of equation (1) has a zero in $[a, b]$.

Proof. Assume, to the contrary, that $y(t)$ is a solution of (1) that has no zeros in $[a, b]$. For definiteness, let $y(t)>0$ on $[a, b]$. Put $r(t)=-y^{\prime}(t) / y(t), t \in[a, b]$. Differentiating $r(t)$ and making use of equation (1), we get

$$
r^{\prime}(t)=a(t) y^{\nu-1}(t)-\frac{g(t)}{y(t)}+r^{2}(t), \quad t \in[a, b],
$$

i.e.

$$
r^{\prime}(t)=a(t) y^{\nu-1}(t)+\frac{|g(t)|}{y(t)}+r^{2}(t), \quad t \in[a, c] .
$$

Using the differential calculus, we see that for $t \in[a, c]$,

$$
\inf _{y>0}\left[a(t) y^{\nu-1}(t)+\frac{|g(t)|}{y}\right] \geq a^{1 / \nu}(t)|g(t)|^{1-1 / \nu} .
$$

This inequality, as well as Hölder's inequality, follows from the equation

$$
x^{\alpha}-\alpha x+\alpha-1 \leq 0, \quad 0<\alpha<1 \text { and } x \geq 0
$$

(cf. p. 12 in [1]). Using this in (2) we get

$$
r^{\prime}(t) \geq a^{1 / \nu}(t)|g(t)|^{1-1 / \nu}+r^{2}(t) .
$$

Multiplying both sides by $u^{2}(t)$ and integrating on $[a, c]$, we obtain, after using integration by parts on the left hand side and the condition $u(a)=u(c)=0$,

$$
0 \geq \int_{a}^{c}\left[a^{1 / \nu}(t)|g(t)|^{1-1 / \nu} u^{2}(t)-{u^{\prime}}^{2}(t)\right] d t+\int_{a}^{c}\left[r(t) u(t)+u^{\prime}(t)\right]^{2} d t .
$$

If the first integral on the right side of this inequality is greater than zero, this gives directly the required contradiction. If the first integral is zero and the second is also zero, this will yield $r(t) u(t)+u^{\prime}(t)=0$. From the definition of $r(t)$, we see that $y(t)$ is a multiple of $u(t)$, i.e. it has zeros at the two points $a$ and $c$ which is again a contradiction with our assumption. In the case of $y(t)<0$ on $[a, b]$, we use the function $z(t)=-y(t)$ as a positive solution of the equation $x^{\prime \prime}(t)+$ $a(t)|x(t)|^{\nu} \operatorname{sgn} x(t)=-g(t)$ and repeat the above procedure on the subinterval $[c, b]$ in place of $[a, c]$. This completes the proof of the theorem. 
Corollary. Assume there exist a sequence of disjoint intervals $\left[a_{n}, b_{n}\right]$, a sequence of points $c_{n} \in\left(a_{n}, b_{n}\right)$, and a sequence of functions $u_{n}(t)$ defined and continuous on $\left[a_{n}, b_{n}\right]$ and differentiable on $\left(a_{n}, c_{n}\right) \cup\left(c_{n}, b_{n}\right)$ with $u_{n}\left(a_{n}\right)=u_{n}\left(c_{n}\right)=u_{n}\left(b_{n}\right)=0$. Let the conditions of the theorem hold, i.e. $a(t)$ is nonnegative on $\left[a_{n}, b_{n}\right]$ and $g(t)$ has different signs on $\left[a_{n}, c_{n}\right],\left[c_{n}, b_{n}\right]$. Then the super-linear equation (1) is oscillatory.

Example. The super-linear equation

$$
x^{\prime \prime}(t)+\beta \sin t|x(t)|^{\nu} \operatorname{sgn} x(t)=\cos t, \quad \nu>1,
$$

is oscillatory if $\beta^{1 / \nu} \geq \pi /\left[2 \frac{\Gamma\left(3+\frac{1}{\nu}\right) \Gamma\left(4+\frac{1}{\nu}\right)}{\Gamma(7)}\right]$, where $\Gamma$ is the gamma function.

Proof. We shall choose

$$
\left[a_{n}, b_{n}\right]=[2 n \pi, 2 n \pi+\pi], \quad c_{n}=2 n \pi+\pi / 2,
$$

and

$$
u_{n}(t)= \begin{cases}\sin \left[\pi\left(t-a_{n}\right) /\left(c_{n}-a_{n}\right)\right] & \text { if } t \in\left[a_{n}, c_{n}\right] \\ \sin \left[\pi\left(t-c_{n}\right) /\left(b_{n}-c_{n}\right)\right] & \text { if } t \in\left[c_{n}, b_{n}\right]\end{cases}
$$

Then

$$
\begin{gathered}
\int_{a_{n}}^{c_{n}}\left[a^{1 / \nu}(t)|g(t)|^{1-1 / \nu} u^{2}(t)-u^{\prime 2}(t)\right] d t \\
=4 \beta^{1 / \nu} \int_{0}^{\pi / 2}\left[\sin ^{2+1 / \nu} t\right]\left[\cos ^{3-1 / \nu} t\right] d t-4 \int_{0}^{\pi / 2} \cos ^{2} 2 t d t \\
=2 \beta^{1 / \nu} \frac{\Gamma\left(3+\frac{1}{\nu}\right) \Gamma\left(4+\frac{1}{\nu}\right)}{\Gamma(7)}-\pi \geq 0 .
\end{gathered}
$$

The same is true for $\left[c_{n}, b_{n}\right]$. Thus, from the theorem, every solution of equation (3) has a zero in $[2 n \pi, 2 n \pi+\pi]$ for every $n$, i.e. the equation is oscillatory.

\section{REFERENCES}

1. E. F. Beckenbach and R. Bellman, Inequalities, "Springer-Verlag", Berlin, 1961. MR 28:1266

2. M. A. El-Sayed, An oscillation criterion for forced second order linear differential equation, Proc. Amer. Math. Soc. 118, 3 (1993), 813-817. MR 93i:34058

3. A. G. Kartsatos, On the maintenance of oscillation of $n$-th order equations under the effect of a small forcing term, J. Diff. Equations 10 (1971), 355-363. MR 44:5556

4. A. G. Kartsatos, Maintenance of oscillations under the effect of a periodic forcing term, Proc. Amer. Math. Soc. 33 (1972), 377-382. MR 48:8959

5. V. Komkov, On boundedness and oscillation of the differential equation $x^{\prime \prime}-A(t) \mathbf{g}(\mathbf{x})=\mathbf{f}(t)$ in $\mathbb{R}^{n}$, SIAM J. Appl. Math. 22 (1972), 561-568. MR 47:554

6. S. M. Rankin, Oscillation theorems for second order nonhomogeneous linear differential equations, J. Math. Anal. Appl. 53 (1976), 550-553. MR 53:6007

7. J. S. W. Wong, Second order nonlinear forced oscillations, SIAM J. Math. Anal. 19, 3 (1988), 667-675. MR 89e:34065

Department of Mathematics, Ain Shans University College for Girls, Asma Fahmi St. Heliopolis, Cairo, Egypt 